\title{
CONCEPT OF RUDRA PASHUPATI AMONG BRAHMINS
}

\section{ब्राह्मणों में रूद्र पशुपति की संकल्पना}

\author{
Dr. Satendra Kumar Mishra 1 网 \\ ${ }^{1}$ Associate Professor, Amity University, Lucknow Uttar Pradesh, India
}

DOI: https://doi.org/10.29121/granthaalayah.v9.i2.2021.3288

Article Type: Research Article

Article Citation: Dr. Satendra Kumar Mishra. (2021). CONCEPT OF RUDRA PASHUPATI AMONG BRAHMINS. International Journal of Research -GRANTHAALAYAH, 9(2), 93-98. https://doi.org/10.29121/granthaalayah.v9.i2.2021.3288

Received Date: 20 January 2021

Accepted Date: 24 February 2021

\section{ABSTRACT}

English : Seven names of Rudra are also mentioned in the Atharvaveda, which appear to be the principal names of Shiva in the later Shaiva sects. These seven names are - bhava, sharava, pashupati, avid, rudra, mahadev, ishaan. Although it is common that all these Namdhari deities are depicted as different deities. Bhava and Shiva are said to be the deity ruling over animals and humans. No human or deity can escape from their arrows. He is invited to kill Yatu Dhanu (demon) and other evil spirits. The body has been called the arrowhead and Bhava has been called the king. A desire has also been expressed to take the deadly poisons of his arrows to another place. The omnipresence of Rudra has also been shown, while he has been described as eminent in fire, in waters, in herbs and other plants, and is said to have created all creatures. It is also known that the same mantra was used for Rudra in Atharvasiras Upanishad and Sweteshvatara Upanishad. It has also been said to be the Bhootpati and Pashupati of Bhava and Sharva. Five types of animals ie cows, blacks, humans and sheep and azas are mentioned under the authority of Pashupati. Rudra is also depicted as Dikpal. Ugra is said to be the lord of the four directions and the sky, earth and space. A Sukta prayed that Rudra should not spread diseases, poisons and heavenly fire. Bhava is called Ish and Rajan. It is said in one place that Bhava, Sharva and Rudra who is Pashupati are always (Sadasiva) welfare. It has also been said that the Gods made the Bhava with arrows the protector of the vratas and the lord of the east direction. Made the body the lord of the south direction. Made Rudra the lord of the earth. Made Mahadev the lord of the divine world. Made Ishan into space.

Hindi : अथर्ववेद में रूद्र के सात नामों का भी उल्लेख किया गया है, जो उत्तरकालीन शैव सम्प्रदायों में शिव के प्रधान नामों के रूप में आते हैं। ये सात नाम हैं - भव, शर्व, पशुपति, उग्र, रूद्र, महादेव, ईशान। यद्यिपि यह सम्य है कि इन सभी नामधारी देवताओं को विभित्र देवताओं के रूप में चित्रित कियाग गया है। भव और शर्व को पशुओं और मानवों पर शासन करने वाला देवता बताया गया है। उनके बाणों से देवता अथवा कोई भी मनुष्य नहीं बच सकता है। उन्हें यातु धानु (राक्षस) तथा अन्य बुरी आत्माओं को मारने के लिए आमन्त्रित किया गया है। शर्व को बाण चलाने वाला कहा गया है और भव को राजा कहा गया है। इस बात की भी इच्छा प्रकट की गई है कि वे अपने बाणों के घातक विषों को दूसरे स्थान पर ले जाएँ। 67 रूद्र ने इन देवताओं को आत्मसात् कर लिया तथापि रूद्र के यही आठ देवरूप पौराणिक शिवके आठ रूपों में समा सकते हैं। रूद्र की सर्वव्यापकता को भी दिखाया गया है, जबकि उसे अग्नि में, जलों में, जड़ी-बूटियों तथा अन्य पौधों में प्रतिष्ट बताया गया है और कहा है कि सभी जीवों को इन्होंने ही बनाया है। यह भी द्रष्टव्य है कि यही मन्त्र अथर्वसिरस उपनिषद और श्वेताश्वेतर उपनिषद् में रूद्र के लिए प्रयुक्त हुआ। भव और शर्व का भूतपति और पशुपति भी कहा गया है।

(C) 2021 The Author(s). This is an open access article distributed under the terms of the Creative Commons Attribution License, which permits unrestricted use, distribution, and reproduction in any medium, provided the original author and source are credited. 
पाँच प्रकार के पशुओं अर्थात् गायों, अश्वों, मनुष्यों और भेड़ों तथा अजों को पशुपति के अधिकार में बताया गया है। रूद्र को दिक्पाल के रूप में भी चित्रित किया गया है। उग्र को चारों दिशाओं तथा आकाश, पृथ्वी एवं अंतरिक्ष का स्वामी बताया गया है। एक सूक्त में प्रार्थना की गई है कि रूद्र व्याधियों, विषों और स्वर्गीय अग्रि को न फैलाए। भव को ईश तथा राजन कहा गया है। एक स्थान में कहा गया है कि भव, शर्व और रूद्र जो पशुपति है, सदैव (सदाशिव) कल्याणकारी हैं। यह भी कहा गया है कि देवताओं ने बाण चलाने वाले भव को व्रात्यों का रक्षक बनाया और पूर्व दिशा का स्वामी बनाया। शर्व को दक्षिण दिशा का स्वामी बनाया। रूद्र को पृथ्वी लोक का स्वामी बनाया। महादेव को दिव्यलोक का स्वामी बनाया। ईशान को अन्तरिक्ष बनाया।

Keywords: ब्राह्मणों; पशुपति

\section{1. प्रस्तावना}

उपर्युक्त विवरण से स्पष्ट है कि अथर्ववेद में रूद्र के भयावह रूप को पूर्ववत रखा गया और उससे मुक्ति पाने के लिए अनेक प्रार्थनायें और अर्चनाये की गई हैं, किन्तु इसके साथ-साथ उसके सदाशिव रूप का विकास भी प्रारम्भ हो गया था। पाशुपत सम्प्रदाय में शिव की जो अष्टमूर्ति की कल्पना है, उसमें से सात नाम तो अथर्ववेद में देखने को मिलते हैं - भव, शर्व, पशुपति, उग्र, रूद्र, महादेव और ईशान। इस प्रकार पाशुपतों ने देव की जो कल्पना की थी, वह इस सृष्टि का कारण है, सर्वव्यापक है, उसका भव बीजरूप में अथर्ववेद में देखने को मिलता है। पाशुपतों की अनेक परम्पराओं का प्रारम्भ बी रूप में अथर्ववेद में हो जाता है।

तैत्तिकीय संहिता तथा अजयमेयि संहिता के शतरूद्रिय में रूद्र्र-षिव के व्यक्तित्व का और अधिक विकास किया गया है। अनेक कल्याणकारी और विनाषकारी रूपों को स्वतन्त्र रूप में देखा गया है। उसे 'गिरीष' अथवा 'गिरित्र' कहा गया है। इसे मार्गों और वनों, पर्वतों का स्वागी कहा गया है और इन स्थानों में घूमनेवाला भी बताया गया है। उसे चोरों तथा समाज बहिष्कृत लोगों का देवता भी बताया गया है। उसके विध्वंसक रूप का प्रकटीकरण अनेक उपाधियों एवं पक्षों से होता है; जैसे किवि, द्रोव्रत्यि। द्रयम्वकहोम के विष्लेषण से भी उसके विध्वंसक स्वरूप पर प्रकाष पड़ता है। अपने ‘भेषज स्वरूप' में उसे स्वर्ग का महान वैद्य कहा गया है। वह पषुओं का भी पति कहा गया है। रूद्र का प्रभाव क्षेत्र इतना व्यापक हो गया था कि उसे दिषाओं का पति भी कहा जाने लगा, उसे 'कपार्देन' भी कहा गया है, जिसका अर्थ है 'जटाजूट' धारण करने वाला। अग्नि से अन्यता के कारण सम्भवतः यह उपाधि उसे मिली थी। शर्व और भय नाम भी उसे दिए गए हैं। इसका भयावह स्वरूप जब सन्तुष्ट हो जाता है तब वह शंकर, शिव और शम्भु हो जाता है। इसे पषुचर्म भी पहने हुए बताया गया है और कृत्रिम वसाननः कहा गया है। सम्भवतः वनों और वनवासियों से सम्बन्धित होने के कारण सम्भवतः उसे पशुचर्म पहनने वाला (कृतिवाआ) कहा गया है। रूद्र को एक भयावह आत्मा के रूप में भी चित्रित किया गया है। उसे गण और गणपति कहा गया है। गणपति नाम महत्वपूर्ण है जो कि यह प्रकट करता है कि रूद्र के व्यक्तित्व में अनेक गणों के देवता की भावना का समावेश हुआ था जो सम्भवतः अनार्य थे। वाजसनेयि सेहिता में तो उसे स्वयं चोर, धोखेबाज आदि की संज्ञा दी गई। यज्जुर्वेद में रूद्र का एक नया नाम त्रयम्बक दिया गया है, जिसके आधार पर पौराणिक षिव के त्रिनेत्र की कल्पना की गई। ऐसा प्रतीत होता है कि यजुर्वेद की रचना के समय तक रूद्र में किसी अनार्य देवता के व्यक्तित्व का समावेष हो गया था जो अनार्य पर्वतवासियों का आराध्य था, इसी कारण से गिरित्र, गिरीष, गिरिचर, गिरिषय, वनानापति कहा गया है। सम्भवतः इस वर्ग के लोग कृषक और वैष्य थे, इसी कारण रूद्र को क्षेत्रपति और वणिक कहा गया है। इसी कारण इसका सम्बन्ध एक स्त्री देवी अम्बिका से किया गया।

ब्राह्मणों में रूद्र पषुपति की संकल्पना में और आगे विकास हुआ। रूद्र-षिव के विध्वंसात्मक स्वरूप का और भी अधिक विकास किया गया। देवता उनसे भयभीत होते हैं। रूद्र की उत्पत्ति सब देवताओं के उग्र वषों के सम्मिश्रण से हुई। घोर और क्रुर उपाधियाँ दी गई। उनके भयावह स्वरूप से लोग इतने त्रस्त थे कि उन्हें अन्य देवताओं से अलग कर दिया गया। शेवगत के साम्प्रदायिक विकास के हष्टिकोण से जो सबसे महत्वपूर्ण प्रवृत्ति ब्राह्मणों में आई वह है रूद्र पाषुपति के अष्ट नामों का विकास। शतपथ ब्राह्मण और कौषीतकि ब्राह्मण में स्पष्ट रूप से कहा गया है कि जन्मोपरान्त, रूद्र को प्रजापति ने आठ नाम दिए, जिनमें सात तो वही हैं जो अथर्ववेद में आते हैं, किन्तु आठवाँ नाम 'अंषभि' है, जिससे अभिप्राय है - रूद्र के वज्रपात से। अथर्ववेद में सातों नामधारी देवताओं को अलग-अलग देवत्व प्रदान किया गया है। किन्तु ब्राह्मणों में इन सभी को पषुपति के विभित्र पक्षों ०े का प्रतीक माना गया है। इनमें रूद्र, शर्व, उग्र और अषनि को प्रकृति के भयावह पक्ष का प्रतीक माना है। भव, 
पषुपति, महादेव और ईषान को प्रकृति के कल्याणगारी तत्वों का प्रतिनिधि माना है। इस प्रकार ब्राह्मण काल में शैवमत की विचारधारा साम्प्रदायिकता की ओर तीव्रगति से बढ़ रही थी, क्योंकि रूद्र षिव की सर्वश्रेष्ठता का सिद्धान्त ब्राह्मणों में कुछ वर्गों में निष्चित रूप से स्वीकार किया जा रहा था।

वेबर महोदय ने बहुत पहले ही कहा था कि कौषीतकि ब्राह्मण में रूद्र को ईषान और महादेव की जो उपाधियाँ दी गई हैं वे स्पष्ट रूप से अन्य देवताओं की तुलना में रूद्र देवता के विषेष महत्ता की ओर संकेत करती है और वस्तुतः साम्प्रदायिक पूजा की ओर भी संकेत करती हैं। आफ्रिच्ट महोदय ने भी यह मत प्रकट किया है कि ब्राह्मणों का काल ही प्राचीन बहुदेववाद के पतन का काल है और शैव मत नामक नवीन धर्म के उत्थान का भ समय है। इन विद्वानों के मतों को हम पूर्णरूपेण तो स्वीकार नहीं कर सकते हैं, क्योंकि ब्राह्मण-काल में साम्प्रदायिक विचारधारा के तत्वों में सर्वश्रेष्ठता तो अवष्य देखने को मिलती है, किन्तु अन्य तत्वों का अभाव है; जैसे - शैव, वैष्णव तथा अन्य साम्प्रदायिक विचारधाराये भक्ति पर आधारित थीं, पूजा उनकी विधि थी। प्रासाद और आत्मसमर्पण उसके प्राण थे। ब्राह्मणों में इन तत्वों का लगभग अभव- सा है। वे तो यज्ञों के वातावरण से आच्छदिंत है।

ब्राह्मणों में रूद्र-षिव अन्य वैदिक देवताओं से पृथक हो गए थे। 'गवेध्युक होम' में कहा गया है कि जिस समय देवगण स्वर्ग गए उस समय रूद्र को पीछे छोड़ दिया, इसलिए उन्हें 'वास्तव्य' (जो घर पर रहे) की उपाधि दी गई। अन्तः में यह भी कहा गया है कि पहले देवताओं ने पषुओं को आपस में विभाजित कर लिया, किन्तु रूद्र का ध्यान नहीं रखा, परन्तु भयभीत होकर उन्हें मूषक समर्पित किया। इसके अतिरिक्त प्रजापति को सरस्वती के प्रति अगम्य गमन के लिए दण्ड देने निमित रूद्र को ही नियुक्त किया गया, क्योंकिवह अन्य देवताओं से पृथक थे। रूद्र की सर्वव्यापकता का सिद्वान्त ब्राह्मणों में उस स्थान पर प्रकट किया गया है, जब रूद्र ने विष्व की प्रत्येक वस्तु को अपना बताया। रूद्र के इस पृथक स्वभाव को कम करने के लिए पुरातन शास्त्र सम्मत वर्ग ने रूद्र और अग्रि के प्राचीन तादात्मय पर पुनः जोर दिया, दूसरे इसकी उत्पत्ति के विषय में अनेक कथाएँ गढ़ी। इतना अवष्य कहा जा सकता है कि शैव धर्म में पाषुपत सम्प्रदाय के आठ नामों की परम्परा का आविर्भाव उस समय हो चुका था।

श्वेताष्वेतर उपनिषद में शैव मत की साम्प्रदायिक पृष्ठभमि पूर्णतया परिलक्षित होती है। रामकृष्ण गोपाल भण्डारकर ने यह मत प्रकट किया है कि उपनिषद् की रचना साम्प्रदायिक भावना को लेकर नहीं हुई है। यद्यपि वह यह स्वीकार करते हैं कि इस उपनिषद् में पूर्ण विकसित रूप में ईष्वरवाद का विकास हुआ, जिसका केन्द्र है एक सात्मक परमेष्वर। उनके मतानुसार 'ष्वेताष्वेतर उपनिषद्' भक्ति परम्परा के द्वार पर खड़ा हुआ है, जिसकी भक्ति का पात्र है रूद्र-शैव। भण्डारकर के मत को पूर्णरूपेण स्वीकार नहीं किया जा सकता है क्योंकि श्रेताष्वेतर उपनिषद् में एक सम्प्रदाय की जितनी विषिष्टतायें होती हैं, वे सभी कुछ प्रच्छत्र रूप से समाविष्ट मिलती है। जैसे रूद्र-षिव की सर्वश्रेष्ठता और सर्वोच्चता। यह तर्क कि उसे केवल देव की उपाधि दी गई है। अतएव यह असाम्प्रदायिक है, उचित नहीं प्रतीत होता, क्योंकि इस देवता का तादात्य रूद्र-षिव, ईषान, महेष्वर से कई बार किया गया है। उसकी श्ति को ‘ईषानी' कहा गया है। उसके समल और निष्कल दोनों रूपों का विवरण प्रस्तत किया गया है। उसे सृष्टि का कारण एवं स्वामी कहा गया है। जो सर्वोंच्य ब्राह्मण है, जो अन्तर्यामी है, जो अग्रि, सूर्य तथा चन्द्र एवं अन्य तत्वों का ही रूप है। महेष्वरको माया का प्रयोग करते हुए बताया गया है। एक स्थान पर कहा गया है कि ईषान जननेन्दिय का अध्यक्ष है, उसी में सभी तत्व सत्रिहित हैं और उसी से सभी वस्तुएं निकलती हैं।षिव जो सृष्ट्रि का कर्ता है, सूक्ष्म से सूक्ष्मतर है, अनेक रूपों वाली है, समस्त सृष्टि में व्याप्त है, उसको जानने से ही मोक्ष मिलता है, उसी में भक्ति करने से मनुष्य मृत्यु के बन्धन से मुक्त हो जाता है।

सांख्य और योग के माध्यम से उसे जाना जा सकता है और अन्त में कहा गया है कि आत्मसमर्पण से मोक्ष मिलता है, क्योंकि इसी परमेष्वर ने ब्राह्मदेव को उत्पन्न किया, इसी ने वेदों को प्रकट किया। संक्षेप में कहा जा सकता है कि श्वेताष्वेतर उपनिषद् में शैवमत की साम्प्रदायिक विचारधारा का उदार रूप से विवरण प्रस्तुत किया गया है। श्वेताष्वेतर उपनिषद् प्राचीन उपनिषदों की परम्परा में आता है। (यद्यपि उसमें सबसे बाद का है।) अतएव उसने अन्य प्राचीन उपनिषदों के सामान्य ज्ञान पर जोर तो दिया, किन्तु भक्ति से अछूता नहीं रह सका। यह द्रष्टव्य है कि पाषुपत सम्प्रदाय में कुछ मूलभूत सिद्धान्त थे; जैसे परमेष्वर का ज्ञानयोग आदि को विषेष स्थान दिया गया है। किन्तु भक्ति आत्मसमर्पण आदि को ही रूद्र सायुज्य का साधन बताया गया है। तुलनात्मक अध्ययन से प्रकट होता है कि श्वेताष्वेतर उपनिषद् के देव और पाषुपत सम्प्रदायों के परमेष्वर की अवधारणा में साम्यता है। ऐसा प्रतीत होता है कि श्वेताष्वेतर उपनिषद् पाषुपत-सम्प्रदाय से अवगत् था, जो समाज में इस समय प्रचलित हो चुका था, किन्तु यह उपनिषद उसका प्रत्यक्ष रूप से उल्लेख नहीं करता है, यद्यपि उसके सिद्धान्तों को उपनिषद 
की विचारधारा में समन्वित करना चाहता है। यह विषेष महत्व की बात है कि उत्तरकाल में पाषुपत-सम्प्रदाय में एक वर्ग ऐसा था, जो वेद सम्मत पाषुपत विचारधारा के आविर्भाव का श्रेय श्रेताचार्य को देता है, जिन्हें यह षिव का उवातार मानता है। यह तथ्य कूर्म पुराण में उल्लिखित है। इस उपनिषद के श्वेताष्वेतर नाम और कूर्म पुराण के श्वेताचार्य में साम्यता महत्वपूर्ण है। यह भी द्रष्टव्य है कि वैदिक साहित्य में रूद्र को जहाँ सदैव रक्तवर्ण कहा गया है, वहाँ उन्हें कभी-कभी श्वेत वर्ण का भी कहा गया है।119यह साम्यतायें आकस्मिक नहीं मानी जासकती हैं। इससे यह अनुमान लगाया जा सकता है कि श्वेताष्वर उपनिषद् जानबूझकर पाषुपत-सम्प्रदाय का प्रत्यक्ष रूप से उल्लेख नहीं करना चाहता था, उसके सिद्धान्तों को अपनाकर वैदिक विचारधारा के शक्ति प्रदान करना चाहता था।

उपर्युक्त विष्लेषण से प्रकट होता है। कि वैदिक साहित्य ने शैव सम्प्रदाय की सैद्धान्तिक पृष्ठभूमि तैयार कर दी थी और इसी पृष्ठभूमि में बैव धर्म के प्रथम सम्प्रदाय पाषुपत का आविर्भाव हुआ। किन्तु पाषुपत सम्प्रदाय तथा शैव-धर्म वैदिक परम्परा की ही एकमात्र फसल नहीं थी, अपितु अनार्य परम्पराओं का भी इस पर प्रचुर प्रभाव पड़ा था, जिसका अवलोकन स्वतन्त्र रूप से वांछनीय है।

\section{2. अनार्य परम्परा का प्रभाव}

शैव धर्म की उत्पत्ति का दूसरा महत्वपूर्ण स्रोत अनार्य परम्परा को माना जा सकता है।

\section{3. सैन्धव सभ्यता का योगदान}

सैन्धव सभ्यता में अनार्य परम्परा की अभिव्यक्ति परिलक्षित होती है। सैन्धव सभ्यता से अनेक ऐसी चित्रकृति शालों पर अंकित है जिन्हें तात्कालिक धार्मिक विचारधारा से सम्बद्ध किया जा सकता है। यद्यपि यह सत्य है कि सैन्धव लिपि का जब तक सन्तोषजनक ढंग से पठन नहीं हो जाता, तब तक निष्चयात्मक रूप से तो कुछ नहीं कहा जा सकता, तथापि प्राप्त साक्ष्यों एवं उपलब्ध विष्लेषणां के आधार पर इतना तो अनुमान लगाया ही जा सकता है कि मातृदेवी के साथ-साथ इस सभ्यता के लोग एक पुरूष देवता के उपासक थे। जिसे मार्षल ने षिव का पूर्व रूप माना है। यह वांछनीय है कि इस देवता के स्वरूप का संक्षेप में विवरण प्रस्तुत किया जाए और यह देखा जाए कि शैव धर्म के पाषुपत-सम्प्रदाय की विचारधारा किसी रूप में इसके प्रच्छन्र है अथवा नहीं है। एक चित्राकृति में इस पुरूष देवता को तीन मुख वाला दिखलाया गया हैं वह एक श्रृंग युक्ल पगड़ी धारण किए हुए पद्मासन मुद्रा में एक सिंहासन पर आसीन है एवं ऊध्वर्तेस् रूप में है और उसके दोनों ओर ऐ व्याघ्र, एक हाथी, एक गैंडा और एक भैंसा प्रदर्षित किया गया है। उसके सिंहासन के नीचे दो हिरण दिखाए गए हैं। यह उपने हाथ में अधिसंख्यक चूड़ियाँ पहने हुए हैं, इसके गले के चारों ओर कवच अथवा उर्षत्राण है। इस चित्रण में अनेक विद्वानों ने शैव-धर्म की तीन विचारधाराएँ देखने का प्रयास किया है। ऐतिहासिक षिव केसमान यह त्रिमुख, पषुपति और योगीष्वर है अथवा महायोगी है। पद्मासन मुद्रा और चंक्षुओं को नासिका के अग्रभाग पर केन्द्रित होने से महायोगी पक्ष पर प्रकाष है। यह भी द्रष्टव्य है कि इस चित्र में कवच षिव के उस रूप का स्मरण दिलाता है, जिसमें वह बाणों से दूसरों का वध करता है, किन्तु स्वयं अजेय है और इस प्रकार उसके सर्वोच्च स्वरूप का उद्घाटन प्रतीकात्मक ढग से किया गया है।

\section{4. निष्कर्ष}

यह बात और स्पष्ट हो जाती है कि जबकि हम उसे दूसरे चित्र में इस पुरुष देवता को एक धनुर्धारी षिकारी के रूप में देखते है। उराका यह रूप वैदिक साहित्य, भहाभारत और पुराण में बहुधा देखने को मिलता है। किरातार्जुनीय में तो षिव इसी रूप में दिखाए गए हैं। उत्तर-पष्चिम भारत में निवास करने वाली अनेक आदिम जातियों जैसे षिव आदि भी षिव को षिकारी रूप में ही देखते थे। पाषुपत-सम्प्रदाय में भी षिव के षिकारी स्वरूप का स्मरण बार-बार किया गया है। यहाँ से अन्य मोहरें भी उपलब्ध हुई है, एक में तो वह लगभग नग्र हैं। केवल कटि प्रदेष में थोड़ा-सा वस्त्र है और सिर पर सींग वाली पगड़ी भी है। एक दूसरी शील में वह पुनः त्रिमुख हैं जबकि दूसरी शील में एक मुख है, किन्तु सींगों के बीच सिर से निकलती हुई पत्तियँँा दिखलाई गई है। भारत के पूर्व ऐतिहासिक कलाओ में कुछ पुरूषाकृतियाँ ऐसी मिली हैं जो धनुर्धर है, सींगोवाली पगाड़ियों से युक्त है 
और पूरे शरीर से अथवा केवल सिर में पत्तियाँपहने हुए हैं। इस चित्रण का प्रतीकात्मक अर्थ यह निकाला जा सकता है कि यह पुरुष देवता प्रकृति की उर्वराषक्ति का प्रतिनिधित्व करता है, यह पक्ष ऊध्वरेतस् मुद्रा से भी प्रकट होता है। यह भी द्रष्टव्य है कि सैन्धव सभ्यता में लिंगोपासना के भी कुछ प्रमाण मिले हैं। कुछ विद्वानों ने ऋग्वेद में आए हुए षिष्नः देवा से अभिप्राय सैन्धववासियों से ही लगाया है, जो लिंगोपासक थे।

ज्ञान की वर्तमान अवस्था में यह कहना कठिन है कि मातृदेवी की इस पुरुष देवता से क्या सम्बन्ध था, किन्तु पाषुपतसम्प्रदाय की उत्पत्ति के हष्टिकोण से ज्ञान की वर्तमान अवस्था में यह कहना कठिन है कि मातृदेवी का इस पुरूष देवता से क्या सम्बन्ध था, किन्तु पाषुपत-सम्प्रदाय की उत्पत्ति के हृष्ट्रिकोण से यह कहा जा सकता है कि सामान्य रूप से शैव सम्प्रदाय और विषेष रूप से पाषुपत-सम्प्रदाय की अनेक प्रवृत्तियों का बीजारोपण यहाँ के आदिवासियों के जीवन में हो गया था और विषेष रूप से सैन्धव सभ्यता में एक पुरूष देवता की पूजा का विधान था जो पाषुपति था, महायोगी था। सृष्टि के क्रियात्मक शक्ति का प्रतीक था, त्रिमुख था। यह सभी प्रवृत्तियाँ पाषुपत-सम्प्रदाय में पायी जाती हैं। आर्यो और अनार्यो के सम्पर्क से वैदिक रुद्र और सैन्धव सभ्यता के महायोगी पषुपति से सामंजस्य हुआ। इसके प्रमाण वैदिक साहित्य, महाभारत तथा पुराणों में देखे जा सकते हैं।

\section{SOURCES OF FUNDING}

None.

\section{CONFLICT OF INTEREST}

None.

\section{ACKNOWLEDGMENT}

None.

\section{REFERENCES}

[1] दि काम्प्रेहिन्सिव ऑफ़ इण्डिया, भाग 2, पृ. 399 ।

[2] पाण्डेय जी.सी., वही, पृ. 301-309।

[3] श्रीवास्तव वी.सी., द एण्टीक्यूटी ऑफ़ दी पाषुपत सेक्ट, के.सी. चठोपाध्याय मेमोरियल वाल्यूम, पृ.124-125, कल्चरल हेरीटेज ऑफ़ इण्डिया, भाग-1, पृ. 389-3991

[4] भण्डारकर आर.जी., वही, पृ. 103

[5] इन विषेषताओं के लिए द्रष्टव्य शतरूद्रिय सुक्त, तैतरिय संहिता, $4 / 5 / 1$ वाजसनेयि संहिता, अध्याय-16।

[6] वाजसनेयि संहिता, $3 / 58$ शतपथ ब्राह्मण 2/6/2/9

[7] यदुवंषी, शैवमत, पृ. 13-17 86. वाजसनेयि संहिता, 3/5 87. यदुवंषी, शैवमत, पृ. 17-20

[8] शतपथ ब्राह्मण, 9/4/1/1-5 पंचविंश ब्राह्मण, 6/9/7, कौषीतकि ब्राह्मण, $3 / 4$

[9] एतरेय, ब्राह्मण, 3/33/1, 3/8/9, शतपथ ब्राह्मण 9/1/1/6/1 तलवाकार ब्राह्मण, 3/26/1।

[10] तैत्तिरीय ब्राह्मण, $3 / 2 / 5$

[11] शतपथ ब्राह्मण $1 / 7 / 3,1-8,9 / 1 / 1 / 5$, तैत्तिरीय ब्राह्मण $1 / 6 / 10$

[12] भण्डारकर, आर.जी., वही, पृ. 108

[13] शतपथ ब्राह्मण, $6 / 1 / 3 / 7$, कौषीतकि ब्राह्मण $6 / 1 / 9$

[14] भण्डारकर, आर.जी., वही, पृ. 105

[15] यदवंषी, घैवमत, पृ. 20, कौषीतकि ब्राह्मण, $23 / 3$ में रूद्र को वेवाधिपति कहा गया है। ईषान ओर महादेव ब्राह्मण ग्रन्थों में उसके सामान्य नाम हैं। रूद्र की श्रेष्ठता ब्राह्मण ग्रन्थों में उल्लिखित दो अन्य कथाओं से भी होती है - (1) प्रजापति की सरस्वती के प्रति गमन की कथा (2) नामोनेधिष्ट की कथा। यह दोनों कथाएँ ऐतरेय ब्राह्मण में क्रमषः $3 / 13 / 9$ और $5 / 22 / 9$ में मिलता है। प्रजापति सरस्वती आख्यान का रूपान्तर जेमिनीय ब्राह्मण $3 / 261 / 63$ में मिलता है। 


\section{डॉ. सतेंद्र कुमार मिश्र}

[16] श्वेताष्वेतर उपनिषद्- $65 / 21$ में कहा गया है कि श्वेताष्वेतर ने ब्रह्म तत्व को

संन्यासियों को बताया।

तपः प्रभावादेवप्रसादाच्च ब्रह्म

ह श्वेताष्वेतरोऽथ विद्वान्।

अत्याश्रमिभ्यः परम पवित्रं

प्रोवाच सम्यगृषिसंधजुषृम्।।

[17] दीवान, पी.सी. लकुलीष ऑफ़ कारवाण एण्ड षिव पाषुपत सेक्ट, जर्नल ऑफ़ दि गुजरात रिसर्च सोसाईटी, भाग (1955) संस्था 4, पृ. 267-2741। इस विद्वान ने ष्वेताष्व को ही पाषुपत-सम्प्रदाय का आदि आचार्य माना ह

[18] संकालिया, एच डी, प्रीहिस्ट्री एण्ड प्रोटोहिस्ट्री ऑफ़ इण्छिया मार्षल, सर जॉन, वही, भाग-1,पृ. 52

[19] पुलाषकार ए.डी. 6 वैदिक ऐज पृ 190, यदुवंषी, शैवमत, अध्याय 2, सुल्लीवान एच.पी., ए रिएक्जामिनेषन ऑफ़ दि रिलीजन ऑफ़ द इण्डस सिविलाइजेषन, दि हिस्ट्री ऑफ़ रिलीजन्स भाग 4, 1 (1964) ने इस पहचान को नहीं माना हैकिन्तु उसके तर्क न्यायसंगत नहीं है। 